\title{
MicroRNA-744 inhibits tumor cell proliferation and invasion of gastric cancer via targeting brain-derived neurotrophic factor
}

\author{
AI-JUN XU ${ }^{1}$, LI-NA FU ${ }^{2}$, HUA-XING WU ${ }^{1}$, XIAO-LI YAO ${ }^{1}$ and RUI MENG ${ }^{1}$ \\ ${ }^{1}$ Department of Endoscope, The Cancer Hospital of Harbin Medical University, Harbin, Heilongjiang 150081; \\ ${ }^{2}$ Department of Gastroenterology, Tianjin Fourth Central Hospital, Tianjin 300140, P.R. China
}

Received March 20, 2017; Accepted July 28, 2017

DOI: $10.3892 / \mathrm{mmr} .2017 .7167$

\begin{abstract}
Gastric cancer is the fourth most common malignancy and the third leading cause of cancer-associated deaths worldwide. It has previously been demonstrated that microRNAs (miRNAs) are actively involved in the pathogenesis of gastric cancer. Therefore, miRNAs have been proposed as promising therapeutic targets in gastric cancer patients. MiR-744 is aberrantly expressed in different types of human cancer. However, the expression pattern and biological roles of miR-744 in gastric cancer remain unknown. The present study demonstrated that miR-744 expression was low in gastric cancer tissues and cell lines. Low expression levels of miR-744 was significantly correlated with lymph node metastasis, invasive depth and TNM staging in gastric cancer patients. The restoration of miR-744 expression inhibited cell proliferation and invasion in vitro. Bioinformatic prediction, luciferase reporter assay, reverse transcription-quantitative polymerase chain reaction and western blot analysis verified that brain-derived neurotrophic factor (BDNF) is a direct target of miR-744 in gastric cancer cells. Furthermore, BDNF was upregulated in gastric cancer tissues and inversely correlated with miR-744 expression. Furthermore, enforced BDNF expression reversed the tumor-suppressing effects of miR-744 on the proliferation and invasion of gastric cancer cells, indicating that BDNF is a functional mediator of miR-744 in gastric cancer. The present study suggests that miR-744 is a potential prognostic biomarker and treatment target in gastric cancer patients.
\end{abstract}

\section{Introduction}

Gastric cancer is the fourth most common malignancy and the third leading cause of cancer-related deaths worldwide (1).

Correspondence to: Professor Hua-Xing Wu, Department of Endoscope, The Cancer Hospital of Harbin Medical University, 150 Haping Road, Nangang, Harbin, Heilongjiang 150081, P.R. China E-mail: hxwu_harbin@163.com

Key words: brain-derived neurotrophic factor, microRNA-744, gastric cancer, proliferation, invasion
Approximately 850,000 newly diagnosed gastric cancer cases and 650,000 deaths are estimated every year (2). Risk factors of gastric cancer include Helicobacter pylori infection, dietary factors, tobacco use and obesity (3). However, the mechanism underlying gastric cancer oncogenesis and progression remains unknown. Despite the considerable improvements in surgical techniques, innovations in clinical diagnostics and development of new chemotherapy regimens, the prognosis of advanced gastric cancer patients remains unsatisfactory, with an overall 5-year survival rate of only $20 \%(4,5)$. Therefore, investigation of the molecular mechanisms underlying the initiation and progression of gastric cancer and development of effective therapeutic strategies for gastric cancer patients are of utmost importance.

MicroRNAs (miRNAs) are 18-22 nt-long endogenous, noncoding and short RNAs which regulate gene expression through complete or partial base pairing with the 3'-untranslated regions (3'-UTRs) of their target genes, resulting in mRNA degradation or translation inhibition (6). Given their effective gene expression regulation, miRNAs modulate various biological processes, such as cell cycle, proliferation, apoptosis, migration, invasion and metastasis $(7,8)$. In recent years, several miRNAs have been confirmed to be aberrantly expressed in various human cancers, such as miR-382 in gastric cancer (9), miR-205 in lung cancer (10), miR-204 in glioma (11) and miR-143 in bladder cancer (12). Many studies have illustrated that deregulated miRNAs can be implicated in tumorigenesis regulation and development by changing their physiological processes (13-15). MiRNAs function as either tumor suppressors or oncogenes depending on the roles of their target genes (16). Tumor-suppressive miRNAs are usually downregulated whereas oncogenic miRNAs are upregulated in cancer (17-19). Therefore, studies on miRNAs are significant in the improvement of human cancer treatments.

miR-744 is aberrantly expressed in different types of human cancer (20-22). However, to the best of our knowledge, studies on miR-744 expression in gastric cancer have yet to be conducted. In this study, we examined miR-744 expression in gastric cancer tissues and cell lines. The association between miR-744 expression and clinicopathological factors was also analysed. Moreover, the roles of miR-744 in gastric cancer and its underlying mechanisms were investigated. 


\section{Materials and methods}

Tissue samples. This study was approved by the Institutional Ethics Committee of the Cancer Hospital of Harbin Medical University. Written informed consent was also obtained from each patient. Fifty-four pairs of gastric cancer tissues and normal gastric tissues were collected from patients with gastric cancer who underwent surgery at the Cancer Hospital of Harbin Medical University between June 2014 and January 2016. None of these patients included in this research received chemotherapy or radiotherapy prior to surgical resection. Upon resection, tissue samples were immediately snap-frozen in liquid nitrogen and then stored at $-80^{\circ} \mathrm{C}$.

Cell lines, culture and transfection. Five human gastric cancer cell lines, including AGS, MKN-45, SGC-7901, BGC-823, and MGC-803, were purchased from Institute of Biochemistry and Cell Biology at the Chinese Academy of Sciences (Shanghai, China). One human gastric epithelial cell line GES-1 were obtained from American Type Culture Collection (Manassas, VA, USA). All cell lines were cultured in Dulbecco's modified Eagle's medium (DMEM) supplemented with $10 \%$ fetal bovine serum (FBS) (both from Gibco; Thermo Fisher Scientific, Waltham, MA, USA), $100 \mathrm{U} / \mathrm{ml}$ penicillin and $100 \mu \mathrm{g} / \mathrm{ml}$ streptomycin in a humidified incubator with 5\% $\mathrm{CO}_{2}$ atmosphere at $37^{\circ} \mathrm{C}$.

miR-744 mimics and negative control miRNA mimics (miR-NC) were bought form GenePharma Co., Ltd. (Shanghai, China). The pCMV6-BDNF plasmid and blank vector(pCMV6) were purchased from OriGene Technologies, Inc. (Rockville, MD, USA). For transfection studies, cells were seeded into 6-well plates at a density of $5 \times 10^{5}$ cells/well. After incubation overnight, cells were transfected with miRNAs or vectors using Lipofectamine 2000 reagent (Invitrogen, Carlsbad, CA, USA) according to the manufacturer's instructions. Then, the plates were incubated in a humidified atmosphere with 5\% $\mathrm{CO}_{2}$ at $37^{\circ} \mathrm{C}$.

Extraction of total RNA and reverse transcription-quantitative polymerase chain reaction $(R T-q P C R)$. Total RNA from tissues or cells was extracted using TRIzol reagent (Thermo Fisher Scientific) according to the manufacturer's protocol. For detection of miR-744, the first-strand cDNA was synthesized using the TaqMan MicroRNA Reverse Transcription kit (Applied Biosystems; Thermo Fisher Scientific). Quantitative PCR (qPCR) was performed on an Applied Biosystems 7500 Sequence Detection system (Thermo Fisher Scientific) using TaqMan MicroRNA PCR kit (Applied Biosystems; Thermo Fisher Scientific), with U6 as an internal control. For detection of brain-derived neurotrophic factor (BDNF) mRNA, total RNA was reverse-transcribed with a PrimeScript RT Reagent kit (Takara Biotechnology Co., Ltd., Dalian, China) and qPCR was conducted with SYBR Premix Ex Taq ${ }^{\mathrm{TM}}$ kit (Takara Biotechnology Co., Ltd.), with GAPDH as an internal control. The sequences of the primers were as follows: miR-744 forward, 5'-AATGCGGGGCTAGGGCTA-3', and reverse, 5'-GTGCAGGGTCCGAGGT-3'; U6 forward, 5'-CGC TTCGGCAGCACATATAC-3' and reverse, 5'-TTCACGAAT TTGCGTGTCAT-3'; BDNF forward, 5'-AGCCTCCTCTTC TCTTTCTGCTGGA-3' and reverse, 5'-TCCCGCCCGACA
TGTCCACT-3'; GAPDH forward, 5'-GACTCATGACCACAG TCCATGC-3' and reverse, 5'-AGAGGCAGGGATGATGTT CTG-3'. Each sample was performed in triplicate and calculated using the $2^{-\Delta \Delta C t}$ method (23).

Cell Counting Kit-8 (CCK-8) assay. Cell proliferation was analyzed with CCK-8 assay (Sigma-Aldrich, St. Louis, MO, USA) according to the manufacturer's instructions. Briefly, cells were plated into 96-well plates at a density of $3 \times 10^{3}$ cells per well. Cells were transfected with miRNAs or vectors. After incubation of $0,24,48$, and $72 \mathrm{~h}, 10 \mu \mathrm{l} \mathrm{CCK}-8$ regent was added into each well and incubated at $37^{\circ} \mathrm{C}$ for additional $2 \mathrm{~h}$. The optical density (OD) at $450 \mathrm{~nm}$ was determined with a multifunction microplate reader (BioTek, Winooski, VT, USA). Each experiment was performed in triplicate and repeated three times.

Transwell cell invasion assay. Cell invasion assay was performed using a Transwell chamber (8.0- $\mu \mathrm{m}$ pores) coated with Matrigel (both from BD Biosciences, San Jose, CA, USA). After transfection $48 \mathrm{~h}$, transfected cells were collected, and a number of $5 \times 10^{4}$ transfected cells were plated on the upper Transwell chambers. DMEM medium containing 10\% FBS was added into the lower chamber as a chemoattractant. Subsequent to $24 \mathrm{~h}$ incubation, the cells remaining in the upper chamber were removed with a cotton swab. The invasive cells were fixed with $100 \%$ methanol and stained with $0.5 \%$ crystal violet. The invasive cells were imaged and counted using an inverted microscope (Olympus Corporation, Tokyo, Japan). Each experiment was repeated at least three times.

Western blot analysis. Total protein was extracted from cells or tissue samples using radioimmunoprecipitation (RIPA) lysis buffer supplemented with protease inhibitor cocktail (Roche Diagnostics, Indianapolis, IN, USA). We detect the protein concentration by using a BCA protein assay kit (Beyotime, Nanjing, China). Subsequently, equal amounts of proteins were separated by $10 \%$ sodium dodecyl sulfate-polyacrylamide gel electrophoresis and transferred to polyvinylidene difluoride membranes (Millipore, Billerica, MA, USA). After that, the membranes were blocked in 5\% nonfat milk in Tris-based saline Tween-20 (TBST) for $1 \mathrm{~h}$ at room temperature, and further incubated overnight at $4^{\circ} \mathrm{C}$ with mouse anti-human BDNF monoclonal antibody (sc-546; 1:1,000 dilution) or mouse anti-human GAPDH monoclonal antibody (sc-47724; 1:1,000 dilution) (both from Santa Cruz Biotechnology, Santa Cruz, CA, USA). After being washed three times for $10 \mathrm{~min}$, the membranes were further incubated with corresponding horseradish peroxidase (HRP)-conjugated secondary antibody (1:5,000 dilution; sc-2005; Santa Cruz Biotechnology) for $1 \mathrm{~h}$ at room temperature. Finally, the protein bands were shown with the application of ECL Immunoblot Detection system (Pierce Biotechnology, Inc., Rockford, IL, USA). GAPDH was used as a loading control, and protein expression was measured using Quantity One software (Bio-Rad, Hercules, CA, USA).

Bioinformatic predication and luciferase reporter assay. The potential targets of miR-744 were analyzed using TargetScan (http://www.targetscan.org/index.html) and and miRanda (http://www.microrna.org/microrna/). 
A

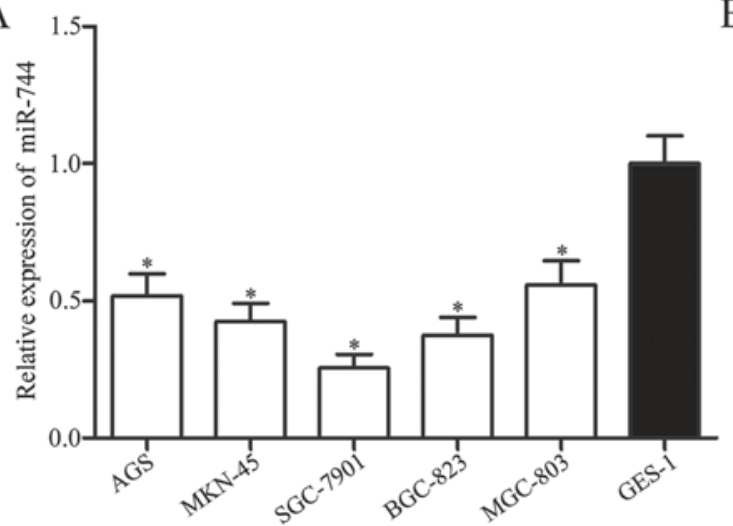

B

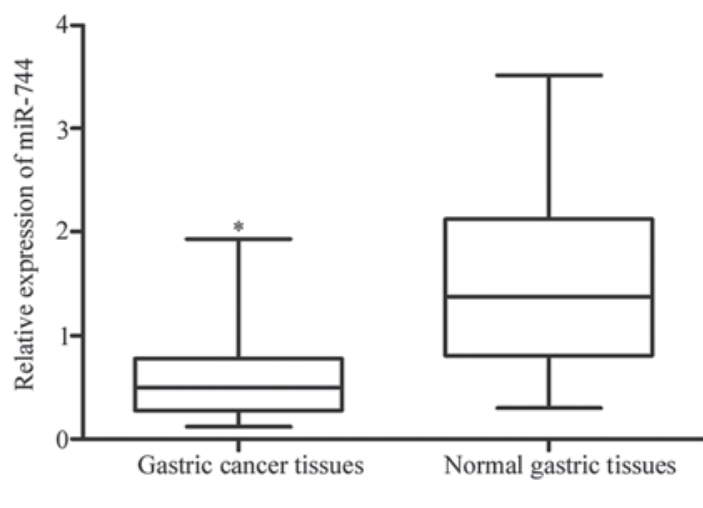

Figure 1. Expression of miR-744 in gastric cancer cell lines and tissue samples. (A) Expression of miR-744 was examined through RT-qPCR in gastric cancer cell lines (AGS, MKN-45, SGC-7901, BGC-823 and MGC-803) and a human gastric epithelial cell line (GES-1). "P<0.05 vs. GES-1. (B) Expression of miR-744 was determined via RT-qPCR in fifty-four pairs of gastric cancer and normal gastric tissues. " $\mathrm{P}<0.05$ vs. normal gastric tissues.

Table I. Relationship between microRNA-744 expression and clinicopathological factors of patients with gastric cancer.

microRNA-744

expression

\begin{tabular}{|c|c|c|c|c|}
\hline $\begin{array}{l}\text { Clinicopathologic } \\
\text { factors }\end{array}$ & $\begin{array}{l}\text { No. of } \\
\text { cases }\end{array}$ & Low & High & $P$-value \\
\hline \multicolumn{5}{|l|}{ Age (years) } \\
\hline$<60$ & 22 & 13 & & \\
\hline$\geq 60$ & 32 & & & \\
\hline \multicolumn{5}{|l|}{ Gender } \\
\hline \multicolumn{5}{|l|}{ Male } \\
\hline \multicolumn{5}{|l|}{ Female } \\
\hline \multicolumn{4}{|l|}{$\underset{<4}{\text { Tumor size }(\mathrm{cm})}$} & 0.599 \\
\hline$<4$ & & 12 & 13 & \\
\hline$\geq 4$ & & 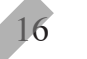 & 13 & \\
\hline \multicolumn{5}{|l|}{ Differentiation } \\
\hline Well and moderat & 50 & 15 & 15 & \\
\hline Poor and signet & 24 & 13 & 11 & \\
\hline \multicolumn{4}{|c|}{ Lymph node metastasis } & $0.030^{\mathrm{a}}$ \\
\hline No & 25 & 9 & 16 & \\
\hline Yes & 29 & 19 & 10 & \\
\hline \multicolumn{4}{|l|}{ Invasive depth } & $0.005^{\mathrm{a}}$ \\
\hline $\mathrm{T} 1+\mathrm{T} 2$ & 31 & 11 & 20 & \\
\hline $\mathrm{T} 3+\mathrm{T} 4$ & 23 & 17 & 6 & \\
\hline \multicolumn{4}{|l|}{ TNM staging } & $0.006^{\mathrm{a}}$ \\
\hline I-II & 21 & 6 & 15 & \\
\hline III-IV & 33 & 22 & 11 & \\
\hline
\end{tabular}

${ }^{\mathrm{a}} \mathrm{P}<0.05$.

For luciferase reporter assays, pMIR-BDNF-3'-UTR wild type $(\mathrm{Wt})$ vector and pMIR-BDNF-3'-UTR mutant (Mut) vector were obtained from GenePharma Co., Ltd.
Cells were seeded into 24-well plates and cotransfected with pMIR-BDNF-3'-UTR Wt or pMIR-BDNF-3'-UTR Mut, and miR-744 mimics or miR-NC using Lipofectamine 2000 reagent. Following incubation at $37^{\circ} \mathrm{C}$ for $48 \mathrm{~h}$, the firefly and Renilla luciferase activities were measured using the Dual-Luciferase Reporter Assay system (Promega, Madison, WI, USA), according to the manufacturer's protocol. Renilla luciferase was chosen as the normalization. Three independent experiments were performed.

Statistical analysis. Data were presented as mean \pm standard deviation, and analyzed using (SPSS) version 18.0 (SPSS Inc., Chicago, IL, USA). The differences between two groups were analyzed using Student's t-test, or assessed by one-way ANOVA when there were more than two groups. A two-tailed value of $P$ less than 0.05 was considered statistically significant.

\section{Results}

Relative miR-744 expression in human gastric cancer and its correlation with clinicopathological characteristics. To determine whether miR-744 expression is associated with gastric cancer, we measured the miR-744 expression of five human gastric cancer cell lines (AGS, MKN-45, SGC-7901, BGC-823 and MGC-803) and that of a human gastric epithelial cell line (GES-1). Results showed that miR-744 was significantly downregulated in the gastric cancer cell lines compared with that in GES-1 (Fig. 1A, P<0.05). In addition, we detected miR-744 expression in fifty-four pairs of gastric cancer tissues and normal gastric tissues. As shown in Fig. 1B, gastric cancer tissues had lower miR-744 expression than normal gastric tissues $(\mathrm{P}<0.05)$.

To clarify the potential clinical significance of miR-744 in gastric cancer, all patients were divided into two groups according to the median miR-744 value: Low-miR-744 group $(\mathrm{n}=28)$ and high-miR-744 group $(\mathrm{n}=26)$. As shown in Table I, low miR-744 expression was significantly correlated with lymph node metastasis $(\mathrm{P}=0.030)$, invasive depth $(\mathrm{P}=0.005)$, and TNM staging $(\mathrm{P}=0.006)$ in gastric cancer patients. However, miR-744 expression was not significantly associated 
A

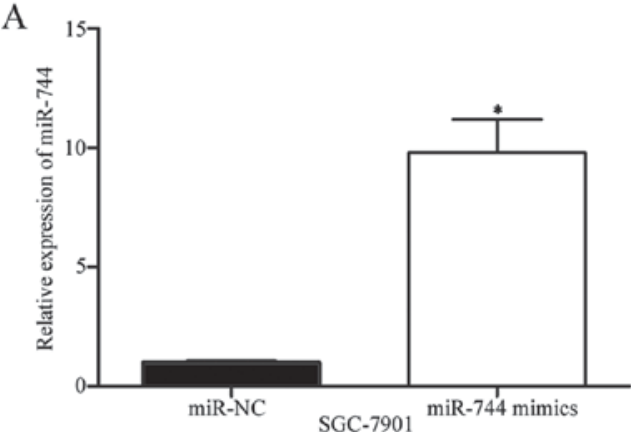

$\mathrm{C}$

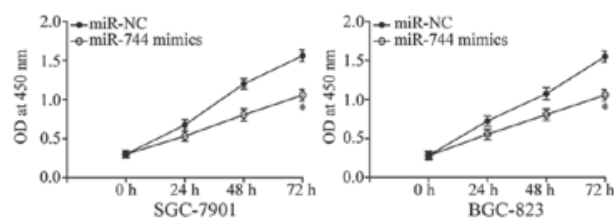

B

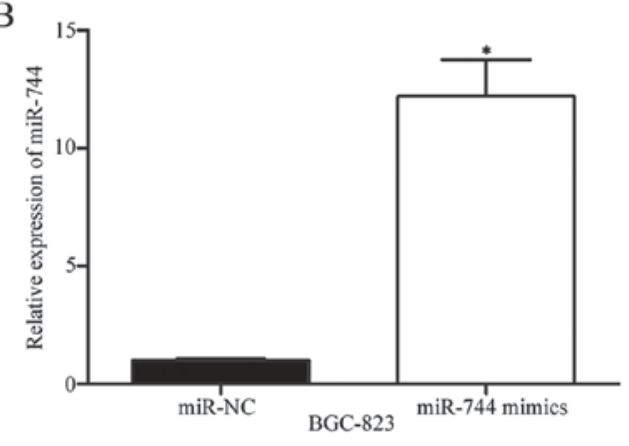

$\mathrm{D}$

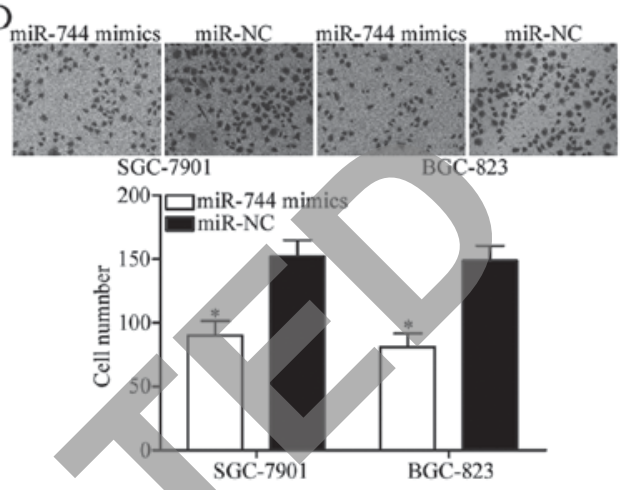

Figure 2. Effect of miR744 on the proliferation and invasion of gastric cancer cells. (A and B) Transfection of miR-744 mimics into SGC-7901 and BGC-823 cells markedly increased miR-744 expression as indicated by the results of RT-qPCR analysis. "P<0.05 vs. miR-NC. (C) CCK-8 assays were utilised to evaluate the effect of miR-744 overexpression on the proliferation of SGC-7901 and BGC-823 cells. "P<0.05 vs. miR-NC. (D) Transwell cell invasion assay was performed to measure the invasiveness of SGC-7901 and BGC-823 cells transfected with miR-744 mimics or miR-NC. " $\mathrm{P}<0.05$ vs. miR-NC.

with age $(\mathrm{P}=0.377)$, gender $(\mathrm{P}=0.607)$, tumor size $(\mathrm{P}=0.599)$ and differentiation $(\mathrm{P}=0.761)$.

Upregulation of miR-744 inhibits the proliferation and invasion of gastric cancer cells. To investigate the biological relevance of miR-744 downregulation on gastric cancer progression, we transfected the gastric cancer cell lines SGC-7901 and BGC-823 with either miR-744 mimics or miR-NC. Transfection efficiency was confirmed in both cell lines using RT-qPCR (Fig. 2A and B, P<0.05). The effect of miR-744 overexpression on gastric cancer cell proliferation was determined using CCK-8 assay. We observed that the proliferation of SGC-7901 and BGC-823 cells was inhibited by miR-744 upregulation compared with that of cells transfected with miR-NC (Fig. 2C). Moreover, Transwell cell invasion assay revealed that the restoration of miR-744 expression repressed the invasion capacities of SGC-7901 and BGC-823 cells (Fig. 2D, P<0.05). These results suggest that miR-744 inhibits the malignant behaviour of gastric cancer cells.

$B D N F$ is a direct target of miR-744 in gastric cancer. To elucidate the underlying mechanism by which miR-744 affects the biological functions of gastric cancer cells, the potential targets of miR-744 were determined using bioinformatic prediction. Among these predicted targets, NKD1 (20), Bcl-2 (24), ARHGAP5 (21), and c-Myc (25) was identified as direct targets. In this study, BDNF was selected for further validation (Fig. 3A) because of its vital role in gastric cancer progression (26). Luciferase reporter assays were conducted to test whether BDNF is a target of miR-744. SGC-7901 and BGC-823 cells were transfected with pMIR-BDNF-3'-UTR wild type (Wt) or pMIR-BDNF-3'-UTR mutant (Mut), along with miR-744 mimics or miR-NC. As shown in Fig. 3B, miR-744 markedly decreased the relative luciferase activities of BDNF-3'-UTR Wt in both SGC-7901 and BGC-823 cells $(\mathrm{P}<0.05)$, whereas the cells transfected with BDNF-3'-UTR Mut did not exhibit decreased luciferase activities in the presence of miR-744. Furthermore, we investigated the regulatory effects of miR-744 on BDNF expression by measuring the mRNA and protein levels of BDNF in SGC-7901 and BGC-823 cells transfected with miR-744 mimics or miR-NC. RT-qPCR and Western blot analyses displayed that miR-744 delivery obviously reduced BDNF expression in SGC-7901 and BGC-823 cells at both mRNA (Fig. 3C, P<0.05) and protein (Fig. 3D, $\mathrm{P}<0.05$ ) levels. Collectively, these results demonstrate that BDNF is a direct target of miR-744 in gastric cancer.

$B D N F$ is upregulated in gastric cancer tissues and negatively correlates with miR-744 expression. We detected BDNF expression in gastric cancer and normal gastric tissues to further explore the association between miR-744 and BDNF in gastric cancer. RT-qPCR and Western blot analyses indicated that BDNF expression was upregulated in gastric cancer tissues compared with normal gastric tissues at both mRNA (Fig. 4A, P<0.05) and protein (Fig. 4B, P<0.05) levels. Spearman's correlation analysis further showed that miR-744 expression negatively correlated with BDNF mRNA expression in gastric cancer tissues (Fig. 4C; $r=-0.6833, \mathrm{P}<0.0001$ ).

BDNF overexpression partially rescued the effects of $\mathrm{miR}-744$ on the proliferation and invasion of gastric cancer cells. After 
A

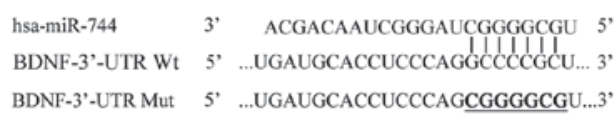

$\mathrm{C}$

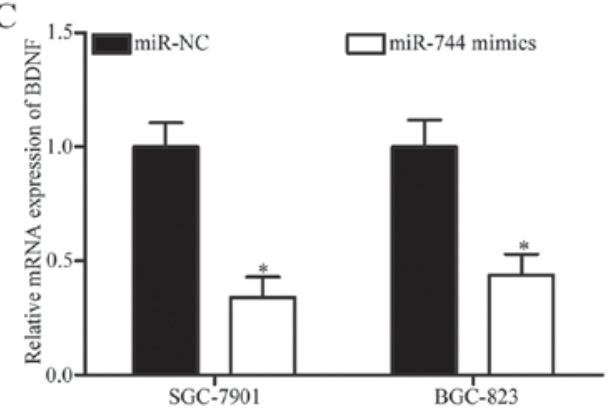

B

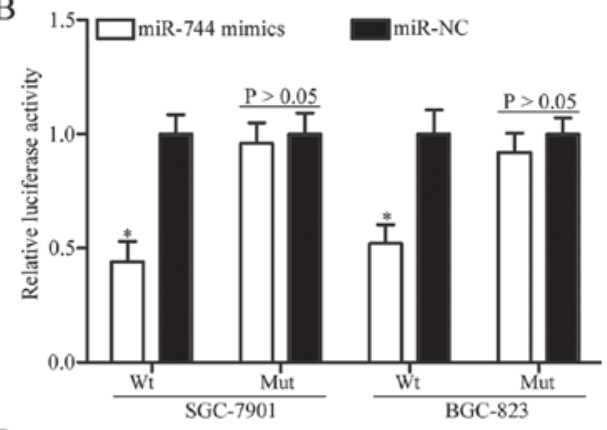

D

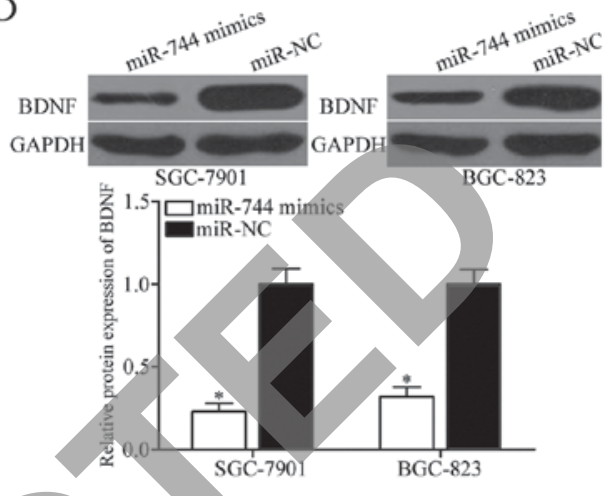

Figure 3. BDNF is a direct target of miR-744 in gastric cancer. (A) Putative binding sites for miR-744 in the 3'-UTR of BDNF were determined using bioinformatic prediction. Mutated sites within the binding sites are shown. (B) Luciferase activities was detected in SGC-7901 and BGC-823 cells co-transfected with reporter plasmids carrying the wild-type (Wt) or mutant (Mut) BDNF 3'-UTR and with miR-744 mimics or miR-NC. "P<0.05 vs. miR-NC. (C) RT-qPCR was used to measure BDNF mRNA expression in SGC-7901 and BGC-823 cells transfected with miR-744 mimics or miR-NC. "P<0.05 vs. miR-NC. (D) Western blot was adopted to detect BDNF protein expression in SGC-7901 and BGC-823 cells transfected with miR-744 mimics or miR-NC. "P<0.05 vs. miR-NC. BDNF, brain-derived neurotrophic factor; CCK-8, Cell Counting Kit-8; 3-UTR, 3'-untranslated region.

identifying BDNF as a direct target of miR-744 in gastric cancer, we next focused on whether BDNF could mediate the biological roles of miR-744 in gastric cancer. SGC-7901 and BGC-823 cells were transfected with miR-NC, miR-744 mimics or miR-744 mimics combined with pCMV6-BDNF. Western blot analysis confirmed that the ectopic expression of miR-744 suppressed BDNF protein expression, whereas co-transfection with pCMV6-BDNF could recover the BDNF expression in SGC-7901 and BGC-823 cells (Fig. 5A, P<0.05). Importantly, the restoration of BDNF expression could reverse the inhibited proliferation (Fig. 5B, $\mathrm{P}<0.05$ ) and invasion (Fig. 5C, P<0.05) imposed by miR-744 in SGC-7901 and BGC-823 cells. These results indicate that miR-744 inhibits the proliferation and invasion of gastric cancer cells, at least in part, through BDNF downregulation.

\section{Discussion}

An increasing number of evidence demonstrated that miRNAs are aberrantly expressed in various types of human cancer (10-12) and actively involved in the pathogenesis of several cancers, including gastric cancer (9). Therefore, miRNAs have been proposed as promising therapeutic targets in gastric cancer patients. In this study, miR-744 was dramatically downregulated in gastric cancer cell lines and tissue samples, suggesting that low miR-744 expression is significantly associated with gastric cancer. Clinicopathological analysis revealed that miR-744 expression negatively correlated with lymph node metastasis, invasive depth and TNM staging in gastric cancer patients. Importantly, miR-744 upregulation suppressed the proliferation and invasion of gastric cancer cells. Furthermore, the effects of miR-744 on gastric cancer cells were at least partly mediated through BDNF suppression. This study is the first to investigate the expression and biological roles of miR-744 in gastric cancer and identify that BDNF is a direct and functional target of miR-744.

The deregulation of miR-744 has been reported in different types of human cancer. For example, miR-744 is downregulated in both hepatocellular carcinoma tissues and cell lines (25). Low miR-744 expression is significantly correlated with microvascular invasion, lymph node metastasis and hepatocellular carcinoma recurrence. In addition, hepatocellular carcinoma patients with low miR-744 expression show obviously worse recurrence-free and overall survival rates than those with high miR-744 expression. Furthermore, multivariate analysis identified low miR-744 expression in hepatocellular carcinoma as an independent predictor of poor prognosis (27). However, miR-744 is upregulated in prostate cancer (20), nasopharyngeal carcinoma (21) and pancreatic cancer (22). In nasopharyngeal carcinoma, high miR-744 expression has been associated with TNM stage, tumorigenesis and metastasis (21). In pancreatic cancer, miR-744 expression in plasma is significantly correlated with lymph node metastasis and disease recurrence (28). These findings suggest that miR-744 expression exhibits tissue specificity and may be a diagnostic and prognostic biomarker for various types of cancer.

Previous studies showed that miR-744 serves as a tumor suppressor in the development of human cancers. Lin et al (25) found that the ectopic expression of miR-744 inhibits cell proliferation and induces G1 accumulation in 
A

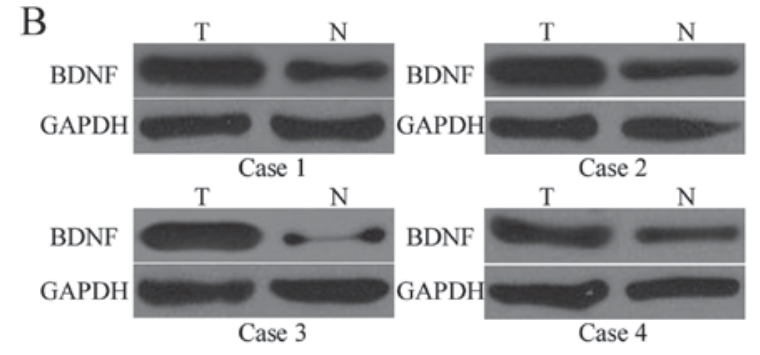

$\mathrm{C}$

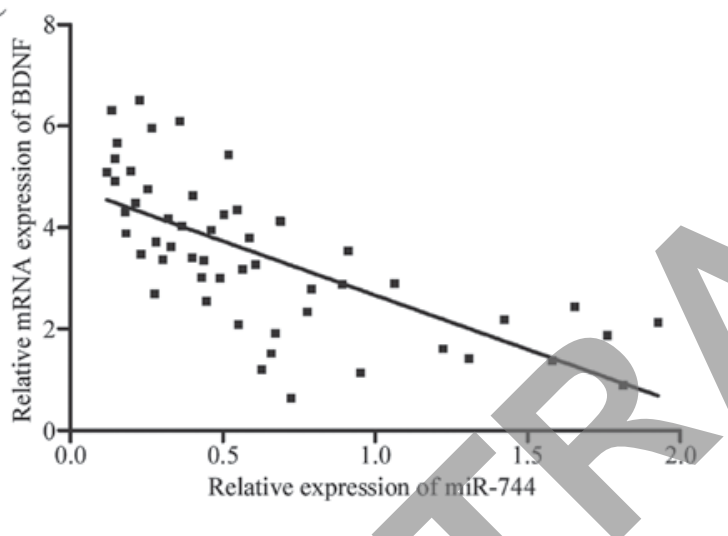

Figure 4. BDNF expression is upregulated in gastric cancer tissues and inversely correlated with miR-744 level. (A) Relative mRNA expression of BDNF was determined through RT-qPCR in gastric cancer and normal gastric tissues. ${ }^{*} \mathrm{P}<0.05$ vs. normal gastric tissues. (B) Western blot analysis of BDNF protein expression in gastric cancer and normal gastric tissues. (C) Spearman's correlation analysis was conducted to explore the correlation between miR-744 and BDNF mRNA expression in gastric cancer tissues. $\mathrm{r}=-0.6833, \mathrm{P}<0.0001$. BDNF, brain-derived neurotrophic factor.

hepatocellular carcinoma. Chen and Liu also reported that miR-744 upregulation decreases cervical cancer cell growth in vitro and in vivo (24). However, miR-744 was identified as an oncogene in prostate cancer because it promotes prostate cancer cell proliferation and metastasis in vitro and reduces tumor growth in vivo (20). Fang et al (21) revealed that the resumption of miR-744 expression increases the cell growth and metastasis of nasopharyngeal carcinoma in vitro and in vivo. Moreover, Zhou et al (22) demonstrated that miR-744 overexpression improves the tumorigenicity and progression of pancreatic cancer. These differences between cancer types may be explained by the 'imperfect complementarity' of the interactions between miRNAs and their target genes (29). These findings also suggest that miR-744 is a promising molecular target for the treatment of such diseases.

BDNF, which is located on the short arm of chromosome 11 (11p13), is an important neurotrophin in the brain (30). Recent
A
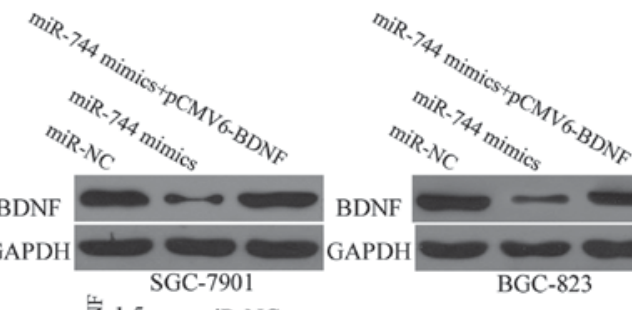

GAPDH

SGC-7901

BDNF

GAPDH

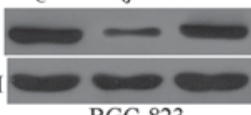

BGC-823

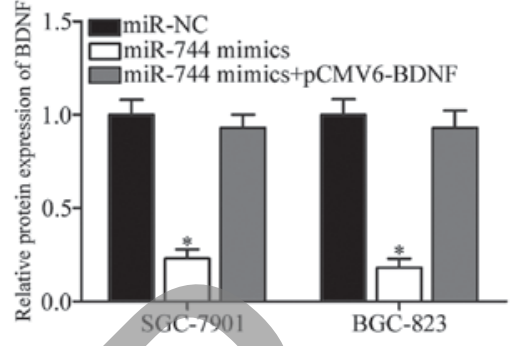

\section{B}

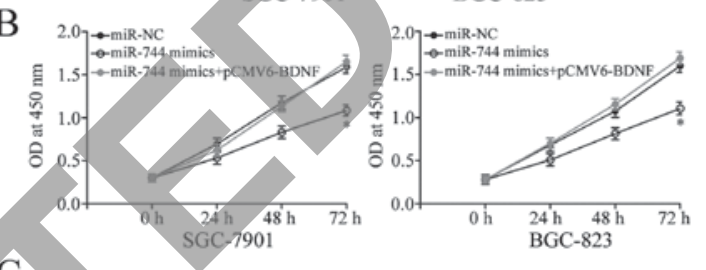

$\mathrm{C}$
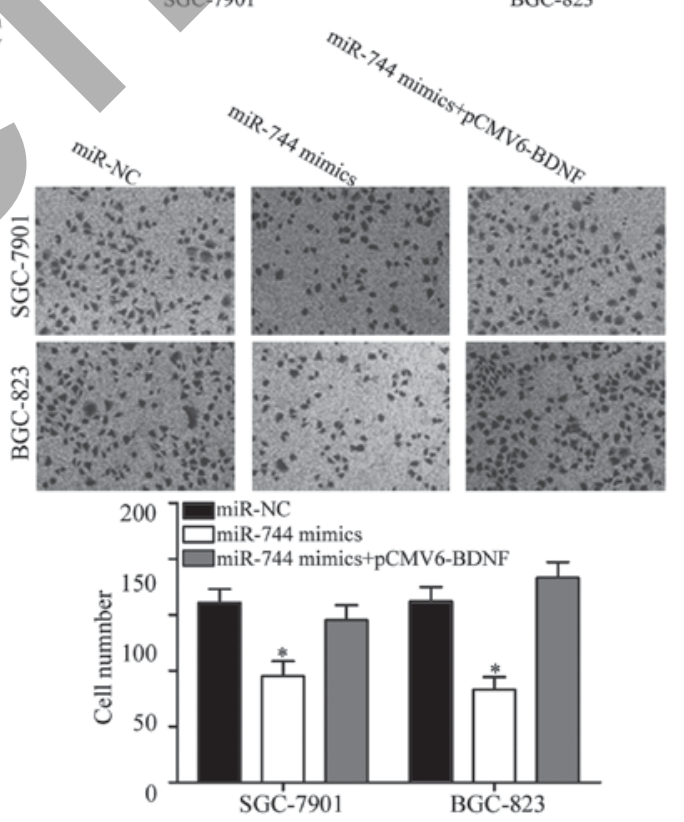

Figure 5. BDNF overexpression partially rescued the miR-744-inhibited proliferation and invasion of gastric cancer cells. (A) Western blot analysis of BDNF protein expression in SGC-7901 and BGC-823 cells co-transfected with miR-NC or miR-744 mimics alone or miR-744 mimics together with pCMV6-BDNF. * $\mathrm{P}<0.05$ vs. miR-NC and miR-744 mimics together with pCMV6-BDNF. (B and C) BDNF upregulation reverted the suppressive effects of miR-744 overexpression on the proliferation and invasion of SGC-7901 and BGC-823 cells. "P<0.05 vs. miR-NC and miR-744 mimics together with pCMV6-BDNF. BDNF, brain-derived neurotrophic factor.

studies have discovered that BDNF is a critical cancer regulator and is abnormally upregulated in several types of human cancer, such as breast (31), bladder (32) and colon (33) cancers and hepatocellular carcinoma (34). Moreover, BDNF is involved in the tumorigenesis and tumor development of several human malignancies (35-37). In gastric cancer, tumor tissues showed higher BDNF expression than adjacent normal gastric mucosa. In addition, high BDNF expression correlates with vessel invasion, lymph node metastasis, peritoneal dissemination and 
poor prognosis in gastric cancer patients $(26,38)$. Functional experiments indicated that BDNF underexpression attenuates the growth and metastasis of gastric cancer cells in vitro and in vivo (26). These findings suggest that BDNF is a therapeutic target in the treatment of gastric cancer.

In conclusion, miR-744, which is downregulated in gastric cancer tissues and cell lines, significantly correlates with lymph node metastasis, invasive depth and TNM staging in gastric cancer patients. Furthermore, miR-744 overexpression suppresses the proliferation and invasion of gastric cancer cells by directly targeting BDNF. Further research exploring the anti-cancer role of miR-744 in gastric cancer may contribute to the development of new therapeutic strategies for patients with this disease.

\section{References}

1. Torre LA, Bray F, Siegel RL, Ferlay J, Lortet-Tieulent J and Jemal A: Global cancer statistics, 2012. CA Cancer J Clin 65: 87-108, 2015.

2. Ferlay J, Shin HR, Bray F, Forman D, Mathers C and Parkin DM: Estimates of worldwide burden of cancer in 2008: GLOBOCAN 2008. Int J Cancer 127: 2893-2917, 2010.

3. Crew KD and Neugut AI: Epidemiology of gastric cancer. World J Gastroenterol 12: 354-362, 2006.

4. Moon YW, Jeung HC, Rha SY, Yoo NC, Roh JK, Noh SH, Kim BS and Chung HC: Changing patterns of prognosticators during 15-year follow-up of advanced gastric cancer after radical gastrectomy and adjuvant chemotherapy: A 15-year follow-up study at a single korean institute. Ann Surg Oncol 14: 2730-2737, 2007.

5. Cunningham D, Allum WH, Stenning SP, Thompson JN Van de Velde CJ, Nicolson M, Scarffe JH, Lofts FJ, Falk SJ, Iveson TJ, et al: Perioperative chemotherapy versus surgery alone for resectable gastroesophageal cancer. N Engl J Med 355: 11-20, 2006.

6. Ambros V: The functions of animal microRNAs. Nature 431: $350-355,2004$.

7. Visone R and Croce CM: MiRNAs and cancer. Am J Pathol 174: $1131-1138,2009$

8. Erson AE and Petty EM: miRNAs and cancer: New research developments and potential clinical applications. Cancer Biol Ther 8: 2317-2322, 2009.

9. Cao Q, Liu F, Ji K, Liu N, He Y, Zhang W and Wang L: MicroRNA-381 inhibits the metastasis of gastric cancer by targeting TMEM16A expression. J Exp Clin Cancer Res 36: 29, 2017.

10. Zeng Y, Zhu J, Shen D, Qin H, Lei Z, Li W, Liu Z and Huang JA: MicroRNA-205 targets SMAD4 in non-small cell lung cancer and promotes lung cancer cell growth in vitro and in vivo. Oncotarget 8: 30817-30829, 2017.

11. Ye ZN, Liu JP, Wu LY, Zhang XS, Zhuang Z, Chen Q, Lu Y, Liu CG, Zhang ZH, Zhang HS, et al: Retraction notice to 'Downregulation of miR-204 expression correlates with poor clinical outcome of glioma patients'. Hum Pathol 63: 46-52, 2017.

12. Wang $\mathrm{H}$, Li Q, Niu X, Wang G, Zheng S, Fu G and Wang Z: miR-143 inhibits bladder cancer cell proliferation and enhances their sensitivity to gemcitabine by repressing IGF-1R signaling. Oncol Lett 13: 435-440, 2017.

13. Xing B and Ren C: Tumor-suppressive miR-99a inhibits cell proliferation via targeting of TNFAIP8 in osteosarcoma cells. Am J Transl Res 8: 1082-1090, 2016.

14. Liu Y, Sun Y and Zhao A: MicroRNA-134 suppresses cell proliferation in gastric cancer cells via targeting of GOLPH3. Oncol Rep 37: 2441-2448, 2017.

15. Zhao Y, Dong Q and Wang E: MicroRNA-320 inhibits invasion and induces apoptosis by targeting CRKL and inhibiting ERK and AKT signaling in gastric cancer cells. Onco Targets Ther 10 1049-1058, 2017.

16. Lee YS and Dutta A: MicroRNAs in cancer. Annu Rev Pathol 4: 199-227, 2009

17. Wu D, Niu X, Pan H, Zhou Y, Zhang Z, Qu P and Zhou J: Tumor-suppressing effects of microRNA-429 in human renal cell carcinoma via the downregulation of Sp1. Oncol Lett 12: 2906-2911, 2016
18. Zhang S, Zhang D, Yi C, Wang Y, Wang $\mathrm{H}$ and Wang J: MicroRNA-22 functions as a tumor suppressor by targeting SIRT1 in renal cell carcinoma. Oncol Rep 35: 559-567, 2016.

19. Ji S, Zhang B, Kong Y, Ma F and Hua Y: miR-326 inhibits gastric cancer cell growth through down regulating NOB1. Oncol Res 25: 853-861, 2017.

20. Guan H, Liu C, Fang F, Huang Y, Tao T, Ling Z, You Z, Han X, Chen S, Xu B and Chen M: MicroRNA-744 promotes prostate cancer progression through aberrantly activating Wnt/ $\beta$-catenin signaling. Oncotarget 8: 14693-14707, 2017.

21. Fang Y, Zhu X, Wang J, Li N, Li D, Sakib N, Sha Z and Song W: MiR-744 functions as a proto-oncogene in nasopharyngeal carcinoma progression and metastasis via transcriptional control of ARHGAP5. Oncotarget 6: 13164-13175, 2015.

22. Zhou W, Li Y, Gou S, Xiong J, Wu H, Wang C, Yan H and Liu T: MiR-744 increases tumorigenicity of pancreatic cancer by activating Wnt/ $\beta$-catenin pathway. Oncotarget 6: 37557-37569, 2015.

23. Livak KJ and Schmittgen TD: Analysis of relative gene expression data using real-time quantitative PCR and the 2(-Delta Delta C(T)) method. Methods 25: 402-408, 2001.

24. Chen XF and Liu Y. MicroRNA-744 inhibited cervical cancer growth and progression through apoptosis induction by regulating Bcl-2. Biomed Pharmacother 81: 379-387, 2016.

25. Lin F, Ding R, Zheng S, Xing D, Hong W, Zhou Z and Shen J: Decrease expression of microRNA-744 promotes cell proliferation by targeting c-Myc in human hepatocellular carcinoma. Cancer Cell Int 14: 58, 2014.

26. Okugawa Y, Tanaka K, Inoue Y, Kawamura M, Kawamoto A, Hiro J, Saigusa S, Toiyama Y, Ohi M, Uchida K, et al: Brain-derived neurotrophic factor/tropomyosin-related kinase $\mathrm{B}$ pathway in gastric cancer. Br J Cancer 108: 121-130, 2013

27. Tan YL, Bai ZG, Zou WL, Ma XM, Wang TT, Guo W, Liu J, Li JS, Jie Yin, Zang YJ and Zhang ZT: miR-744 is a potential prognostic marker in patients with hepatocellular carcinoma. Clin Res Hepatol Gastroenterol 39: 359-365, 2015.

28. Miyamae M, Komatsu S, Ichikawa D, Kawaguchi T, Hirajima S, Okajima W, Ohashi T, Imamura T, Konishi H, Shiozaki A, et al: Plasma microRNA profiles: Identification of miR-744 as a novel diagnostic and prognostic biomarker in pancreatic cancer. Br J Cancer 113: 1467-1476, 2015.

29. Wei C, Luo Q, Sun X, Li D, Song H, Li X, Song J, Hua K and Fang L: MicroRNA-497 induces cell apoptosis by negatively regulating $\mathrm{Bcl}-2$ protein expression at the posttranscriptional level in human breast cancer. Int J Clin Exp Pathol 8: 7729-7739, 2015.

30. Barde YA, Edgar D and Thoenen H: Purification of a new neurotrophic factor from mammalian brain. EMBO J 1: 549-553, 1982.

31. Patani N, Jiang WG and Mokbel K: Brain-derived neurotrophic factor expression predicts adverse pathological and clinical outcomes in human breast cancer. Cancer Cell Int 11: 23, 2011.

32. Lai PC, Chiu TH and Huang YT: Overexpression of BDNF and TrkB in human bladder cancer specimens. Oncol Rep 24: 1265-1270, 2010.

33. Yang X, Martin TA and Jiang WG: Biological influence of brain-derived neurotrophic factor (BDNF) on colon cancer cells. Exp Ther Med 6: 1475-1481, 2013.

34. Yang ZF, Ho DW, Lam CT, Luk JM, Lum CT, Yu WC, Poon RT and Fan ST: Identification of brain-derived neurotrophic factor as a novel functional protein in hepatocellular carcinoma. Cancer Res 65: 219-225, 2005

35. Kang HJ, Kim JM, Kim SY, Kim SW, Shin IS, Kim HR, Park MH, Shin MG, Yoon JH and Yoon JS: A longitudinal study of BDNF promoter methylation and depression in breast cancer. Psychiatry Investig 12: 523-531, 2015.

36. Kaplan DR, Matsumoto K, Lucarelli E and Thiele CJ: Induction of TrkB by retinoic acid mediates biologic responsiveness to BDNF and differentiation of human neuroblastoma cells. Eukaryotic Signal Transduction Group. Neuron 11: 321-331, 1993.

37. Tanaka K, Okugawa Y, Toiyama Y, Inoue Y, Saigusa S, Kawamura M, Araki T, Uchida K, Mohri Y and Kusunoki M: Brain-derived neurotrophic factor (BDNF)-induced tropomyosin-related kinase B (Trk B) signaling is a potential therapeutic target for peritoneal carcinomatosis arising from colorectal cancer. PLoS One 9: e96410, 2014.

38. Choi B, Lee EJ, Shin MK, Park YS, Ryu MH, Kim SM, Kim EY, Lee HK and Chang EJ: Upregulation of brain-derived neurotrophic factor in advanced gastric cancer contributes to bone metastatic osteolysis by inducing long pentraxin 3 . Oncotarget 7 : 55506-55517, 2016 